\title{
Idade Materna como Fator de Risco para a Hipertensão Induzida pela Gravidez: Análise Multivariada
}

\author{
Maternal Age as a Risk Factor for Pregnancy-induced Hypertension: Multivariate Analysis
}

Hélio de Lima Ferreira Fernandes Costa, Cícero Ferreira Fernandes Costa, Laura Olinda Bregieiro Fernandes Costa

\begin{abstract}
RESUMO
Objetivo: verificar se a idade materna igual ou superior a 40 anos é fator de risco independente para o surgimento da hipertensão induzida pela gravidez (HIG).

Método: foi realizado estudo tipo coorte retrospectivo, envolvendo a revisão dos prontuários médicos de 2047 parturientes, das quais 298 com idade igual ou superior a 40 anos e 1749 com idade inferior a 40 anos. Foi realizada análise de regressão logística múltipla para testar a associação da idade materna com a ocorrência de HIG, ajustando o resultado pela paridade, presença de hipertensão arterial crônica, diabete e gemelidade.

Resultados: entre as pacientes com idade igual ou superior a 40 anos a incidencia de HIG foi de 22,1\% (66/298), superior à das pacientes com idade inferior a 40 anos (16\%, 286/ 1463). A HIG foi diagnosticada em $27,2 \%$ das primigestas (174/640), 47,6\% das hipertensas crônicas (30/66) e 27,1\% das diabéticas (13/48). A idade materna avançada, a primiparidade e a hipertensão arterial crônica estiveram associadas à ocorrência de HIG na análise bivariada com OR de 1,4, 2,58 e 4,69, respectivamente. Houve tendência estatisticamente não significante de associação com o diabete gestacional. Após o ajuste, observou-se aumento da força da associação da idade materna avançada com a HIG (OR ajustado = 1,69), o mesmo se verificando em relação à paridade e à hipertensão arterial.

Conclusões: a idade materna igual ou superior a 40 anos foi fator de risco para o surgimento da HIG independente da paridade e da presença da hipertensão arterial e do diabete.
\end{abstract}

PALAVRAS-CHAVE: Hipertensão induzida pela gravidez. Idade materna. Hipertensão. Diabete melito.

\section{Introdução}

A hipertensão arterial que se inicia e termina na gravidez é um grande enigma da obstetrícia. Não se conhece a sua etiologia, apesar de tantos estudos existentes na busca de desvendá$1 \mathrm{a}^{1}$. Alguns fatores de risco parecem definidos, como é o caso da primiparidade, enquanto outros permanecem aguardando definição. A idade mater-

Disciplina de Tocoginecologia, Faculdade de Ciências Médicas, Universidade de Pernambuco (UPE)

Correspondência:

Hélio de Lima F Fernandes Costa

Rua Bruno Maia, 217, Ap 1001 - Graças

52011-110 - Recife - PE na é um deles, havendo controvérsia se a gravidez nos extremos do periodo procriativo eleva os riscos das sindromes hipertensivas.

A definição dos riscos gestacionais inerentes à gestação em idade avançada tem sido preocupação da obstetrícia, uma vez que cresce o número de mulheres em todo o mundo que retardam a primeira gravidez. A busca pela independência financeira, o maior tempo de formação requerido pelo mercado de trabalho moderno, as separações e novas uniões conjugais, aliados às possibilidades oferecidas pelos atuais métodos de anticoncepção e fertilização assistida contribuem para este fenômeno universal ${ }^{2,3}$.

Permanece controversa a associação da idade materna avançada com o desencadeamento da 
hipertensão induzida pela gravidez (HIG). Estudo multicêntrico norte-americano do The Calcium for Preeclampsia Prevention (CPEP) Study Group ${ }^{4}$ não encontrou variação na incidência de HIG nas diversas faixas etárias, ao passo que outros estudos de casuísticas volumosas ${ }^{5,6}$ apontam maior incidência nas gestantes mais idosas. van Katwijk e Peeters ${ }^{7}$, em revisão da literatura, atribuem a maior incidência de HIG na idade avançada à elevada ocorrência, nesta faixa etária, de hipertensão crônica e diabete, questionando o papel da idade como fator de risco independente para o surgimento da HIG.

O presente estudo objetiva verificar se a idade materna igual ou superior a 40 anos é fator de risco independente para o desenvolvimento da HIG, controlando os resultados pela paridade, hipertensão arterial crônica, gemelidade e diabete mélito.

\section{Pacientes e Métodos}

Foi realizado estudo do tipo coorte retrospectivo na Maternidade da Encruzilhada do Centro Integrado de Saúde Amaury de Medeiros (CISAM) - Maternidade-Escola da Faculdade de Ciências Médicas da Universidade de Pernambuco, no período compreendido entre junho de 1999 e agosto do ano 2000.

Foi considerado como critério de inclusão ter sido atendida na Clínica Obstétrica da Maternidade da Encruzilhada para resolução da gravidez, entre $1^{\circ}$ de janeiro de 1994 e 28 de março de 1999 . Como critérios de exclusão foram considerados: idade gestacional igual ou inferior a 20 semanas ou peso fetal inferior a $500 \mathrm{~g}$, extravio total ou parcial do prontuário médico ou preenchimento incompleto do prontuário de forma a impossibilitar a coleta dos dados requeridos para o estudo.

Os dados foram obtidos a partir do Registro de Admissão e Alta e dos prontuários médicos do Serviço de Arquivo Médico e Estatística. Foi estabelecida proporção de 1:5 entre casos e controles. Assim, todas as pacientes com idade igual ou superior a 40 anos foram selecionadas, bem como as cinco pacientes seguintes com idade inferior a 40, anos segundo a ordem dos registros na instituição. Após a seleção dos prontuários e a aplicação dos critérios de exclusão foram analisados os registros de 2047 pacientes, das quais $298 \mathrm{com}$ idade igual ou superior a 40 anos e 1749 com idade inferior a 40 anos.

A ocorrência de HIG foi a variável dependente, sendo considerada como presente se o seu diagnóstico constasse do prontuário da parturiente ou se houvesse o registro de pressão arterial igual ou superior a 140 × $90 \mathrm{mmHg}$ em pelo menos duas oportunidades distanciadas de pelo menos quatro horas em gestante previamente normotensa ou incremento de $30 \mathrm{mmHg}$ na pressão sistólica e/ ou $15 \mathrm{mmHg}$ na pressão diastólica em paciente previamente hipertensa, com ou sem proteinúria. Foram estudadas como variáveis de controle: a paridade, a hipertensão arterial crônica, o diabete melito clínico ou gestacional e a gemelidade. As variáveis de controle foram definidas conforme os diagnósticos registrados no prontuário e revisados no momento da coleta de dados, de acordo com o manual de normas do serviço vigente à época. Assim, hipertensão arterial crônica foi definida como pressão arterial igual ou superior a $140 \times 90$ $\mathrm{mmHg}$ registrada em pelo menos duas oportunidades distintas no prontuário da paciente fora da gravidez ou na primeira metade desta. Diabete melito foi diagnosticado de acordo com os critérios de Bertini Oliveira et $a .^{8}$, adotados no serviço na época da ocorrência dos partos.

Foi elaborada ficha especial para a coleta de dados que interessavam à pesquisa, os quais foram, em seguida, inseridos e manipulados nos aplicativos de domínio público Epi-Info 6.04 do CDC-EUA e MULTLR. Inicialmente foi realizada análise bivariada testando a associação da variável independente (idade) e de cada variável confundidora (paridade, hipertensão arterial crônica, diabete e gemelidade) com a variável de desfecho (HIG) pelo cálculo da odds ratio (OR) e do valor de p. Em seguida foi realizada regressão logística múltipla não condicional pelo método de máxima verossimilhança. O modelo foi saturado progressivamente com a adição de cada uma das variáveis, observando-se, para fins de inclusão no modelo definitivo, seus efeitos sobre a precisão do mesmo e sobre o ajuste das demais variáveis.

O presente estudo foi aprovado pelo Comitê de Ética em Pesquisa do CISAM.

\section{Resultados}

A incidência de HIG nas pacientes com idade igual ou superior a 40 anos foi de $22,1 \%$, significativamente superior àquela observada nas pacientes mais jovens $(16,4 \%)$, com OR de 1,46 (IC = $1,06$ a 1,99$)$. A HIG foi diagnosticada em $27,2 \%$ das primigestas (174/640), 47,6\% das gestantes hipertensas crônicas $(30 / 66)$ e $27,1 \%$ das diabéticas $(13 / 48)$. A primiparidade e a hipertensão arterial crônica também constituíram fatores de ris- 
co para a ocorrência de HIG na análise bivariada, observando-se risco de 2,58 (IC $=2,02$ a 3,29) vezes maior de HIG nas primigestas e 4,69 (IC $=2,73$ a 8,07 ) vezes maior nas gestantes hipertensas. A ocorrência do diabete melito clínico ou gestacional apresentou OR de 1,82 , porém com intervalo de confiança passando pela unidade $(0,90$ a 3,63).

A gemelidade não constituiu na nossa amostra fator de risco estatisticamente significante para a ocorrência de HIG $(\mathrm{p}=0,5)$. Houve apenas 2 casos de HIG em 17 gestações de gêmeos $(11,8 \%)$. Pela falta de associação com a HIG e pela baixa freqüência na amostra, a gemelidade não foi incluída no modelo multivariado.

$\mathrm{Na}$ análise multivariada, a idade, paridade e hipertensão arterial crônica mantiveram associação estatisticamente significante com a ocorrência de HIG. Após o ajuste pela demais variáveis, observamos que houve incremento na força da associação de cada uma das três variáveis mencionadas com a incidência de HIG. De fato, o OR ajustado foi superior ao OR não ajustado para as três variáveis mencionadas: $(1,69 \mathrm{x}$ 1,46 para idade igual ou superior a 40 anos; 3,29 x 2,58 para primiparidade; $5,67 \times 4,69$ para hipertensão arterial crônica).

A probabilidade de ocorrência de HIG poderia ser definida em função das variáveis analisadas como:

$\mathrm{P}=1 / 1+\exp -\left(-2,19+0,52 *\right.$ idade $340 \mathrm{a}+1,19^{*}$ gesta $=1+$ 1,73 *hipertensão crônica), atribuindo-se para as variáveis valor "1" quando a condição estiver presente e valor "0" quando a condição estiver ausente. Os resultados encontram-se resumidos na Tabela 1.

Tabela 1 - Distribuição de 2047 parturientes segundo a ocorrência de DHEG e de seus supostos fatores de risco: idade, hipertensão crônica, diabete e gemelidade. Maternidade da Encruzilhada, Recife (janeiro 1994-março 1999).

\begin{tabular}{|c|c|c|c|c|c|c|c|c|}
\hline \multirow[b]{2}{*}{ Fator de risco } & \multicolumn{2}{|c|}{ HIG } & \multirow[b]{2}{*}{$\mathrm{T}$} & \multirow[b]{2}{*}{ OR (IC) } & \multicolumn{4}{|c|}{ Análise Multivariada } \\
\hline & $\underset{\mathrm{n}(\%)}{\operatorname{Sim}}$ & $\begin{array}{l}\text { Não } \\
\text { n (\%) }\end{array}$ & & & $\mathrm{p}$ & $\beta$ & OR ajustado (IC) & $\mathrm{p}$ \\
\hline \multicolumn{9}{|l|}{ Idade } \\
\hline$\geq 40 \mathrm{a}$ & $66(22.1)$ & $232(77.9)$ & 298 & $1.46(1.06-1.99) 0.014$ & & 0,52 & $1.69(1.19-2.40)$ & 0.03 \\
\hline$\leq 39 a$ & $286(16.4)$ & $1463(83.6)$ & 1749 & 1 & & & & \\
\hline \multicolumn{9}{|l|}{ Paridade } \\
\hline 1 & $174(27.2)$ & $466(72.8)$ & 640 & $2.58(2.02-3.29)<0.001$ & & 1,19 & $3.29(2.55-4.24)$ & $<0.001$ \\
\hline $2 \mathrm{ou}+$ & $178(12.7)$ & $1229(87,3)$ & 1407 & 1 & & & & \\
\hline \multicolumn{9}{|l|}{ H.A.C } \\
\hline Sim & $30(47,6)$ & $33(52,4)$ & 66 & $4,69(2,73-8,07)<0,001$ & $<0,001$ & & $1,735,67(3,28-9,80)$ & $<0,001$ \\
\hline Não & $322(16,2)$ & $1662(83,8)$ & 1984 & 1 & & & & \\
\hline \multicolumn{9}{|l|}{ Diabete Mélito } \\
\hline Sim & $13(27,1)$ & $35(72,9)$ & 48 & $1,82(0,90-3,63) 0,06$ & 0,06 & & Não incluída no modelo & \\
\hline Não & $339(17,0)$ & $1660(83,0)$ & 1999 & 1 & & & & \\
\hline \multicolumn{9}{|l|}{ Gemelidade } \\
\hline Sim & $2(11,8)$ & $15(88,2)$ & 17 & $0,64(0,10-2,97) 0,5$ & 0,5 & & Não incluída no modelo & \\
\hline Não & $350(17,2)$ & $1680(82,8)$ & 2030 & 1 & & & & \\
\hline Total & $352(17,2)$ & $1695(82,8)$ & 2047 & & & & & \\
\hline
\end{tabular}

HAC = hipertensão arterial crônica; HIG = hipertensão induzida pela gravidez.

$\beta=$ coeficiente de regressão

$\mathrm{T}=$ total

$\mathrm{OR}=$ odds ratio

IC = intervalo de confiança

\section{Discussão}

Não há padronização entre os diversos autores a respeito do limite inferior da idade considerada tardia para engravidar. A maioria dos estu- dos estabelece o ponto de corte entre 30 e 40 anos $^{9}$, havendo, entretanto, os que a fixam em 50 anos $^{10}$. Além da dificuldade em estatuir o aludido limite há o problema referente à escolha do grupo controle. Qual seria o ideal? A faixa de maior nubilidade, isto é, de 20 a 29 anos, ou considerar 
todo o grupo com idade inferior ao investigado? Optamos por definir o grupo de casos a partir dos 40 anos, por ser o ponto de corte mais utilizado na literatura, e o grupo controle como as não portadoras da condição considerada de risco, ou seja, as gestantes com idade abaixo dos 40 anos.

A incidência da HIG no grupo com idade igual ou superior a 40 anos foi elevada e significativamente maior do que aquela observada no grupo controle $(22,1 \%$ x $16,4 \%)$. Ziadeh e Yahaya ${ }^{5}$ apresentam resultados semelhantes aos nossos e até com casuística mais encorpada, representada por 468 pacientes com idade igual ou maior de 40 anos. Entretanto, escolheram como grupo controle mulheres com idade entre 20 e 29 anos, isto é, a faixa etária em que a nubilidade é mais expressiva. Desta forma, poderiam estar superestimando o pior prognóstico das gestantes de 40 anos ou mais, ao compará-lo com o grupo que, teoricamente, teria os melhores resultados obstétricos, e não com a população de grávidas abaixo dos 40 anos.

$\mathrm{Na}$ análise bivariada, além da idade, também a hipertensão arterial crônica e a primiparidade revelaram-se como fatores de risco importantes para o surgimento da HIG, ao passo que o diabete apenas apresentou tendência, próxima do limite de significância, a maior incidência de HIG. Esses resultados são concordantes com conceitos bem estabelecidos na literatura ${ }^{11,12}$ de serem, a primiparidade e a hipertensão arterial crônica, fatores de risco para o surgimento da HIG. O pequeno número de gestações de gêmeos na amostra não permite conclusões acerca de sua associação com a variável de desfecho.

O risco não ajustado do desenvolvimento de HIG no grupo de gestantes idosas foi cerca de 50\% maior que no grupo mais jovem. Embora a análise bivariada exprima o risco real das pacientes com idade igual ou acima dos 40 anos, seu valor para explicar o fenômeno biológico é limitado, uma vez que o efeito da idade não pode ser isolado do efeito de outras variáveis que se modificam de acordo com o avançar da idade . $^{7}$

Após o ajuste pelas variáveis de confundimento observou-se incremento da força da associação da idade avançada com a ocorrência da HIG, com elevação do OR de 1,46 para 1,69. Provavelmente a paridade atuava na análise bivariada como fator de confundimento, subestimando o efeito da idade na determinação da HIG, visto que na idade avançada encontra-se maior proporção de multíparas, que é fator de proteção para a variável de desfecho. Por outro lado, a hipertensão arterial na análise bivariada tende a superestimar a associação entre idade e HIG, visto que a hipertensão se associa positivamente com ambas as variá- veis. Portanto, nossos resultados revelam que o efeito do ajuste pela paridade superou o efeito do ajuste pela hipertensão crônica, uma vez que o efeito líquido do ajuste final foi o aumento do OR. Podemos afirmar, desta forma, que a maior freqüência de multíparas entre as grávidas de 40 anos ou mais tendeu a subestimar a incidência de HIG. Ademais, a magnitude deste efeito de confundimento superou a superestimação da incidência de HIG esperada pela maior ocorrência de hipertensão arterial crônica nessa faixa etária.

A presença de diabete foi testada na análise de regressão logística, porém não permaneceu no modelo final pelo fato de não alterar a significância do mesmo e não ajustar significativamente o OR das variáveis já inseridas. A ausência de significância quanto à associação entre o diabete e a HIG foi resultado inesperado. Sendo o nosso estudo retrospectivo e o diabete gestacional entidade diagnosticável apenas por testes de tolerância à glicose, muitas vezes não incluídos na rotina prénatal dos serviços da região, é provável que um subdiagnóstico do diabete possa ter mascarado seu verdadeiro significado em relação à HIG. Contrariamente aos nossos achados, associação entre o diabete melito e a hipertensão arterial, seja ela crônica ou induzida pela gravidez, tem sido bem demonstrada em diversos estudos ${ }^{13,14}$.

Seoud et al. ${ }^{15}$, em análise bivariada estratificada de 319 gestantes com 40 ou mais anos e 326 controles, encontraram maior ocorrência de HIG em gestantes idosas e multíparas, mas não entre as nulíparas. A análise estratificada, embora tenha a vantagem de estimar o risco de grupos específicos, reduz o número de casos nos diferentes estratos e, portanto, pode não ter tido, no estudo em questão, o poder de identificar diferenças entre as nuligestas, grupo com alta incidência de HIG em todas as faixas etárias.

Hrazdilova et al. ${ }^{16}$ não encontraram, analisando por regressão logística um grupo de 910 parturientes, associação independente entre a préeclâmpsia e a idade materna, mas sim com o índice de massa corporal. Seu grupo de estudo, entretanto, tinha média de idade de 27 anos, portanto, não era capaz de aferir o efeito das idades extremas (mais de 40 anos).

Nossos dados reforçam o conceito de que a idade avançada aumenta o risco de HIG, independente da paridade, presença de hipertensão crônica ou diabete. É possivel que o comprometimento vascular inerente à idade torne algumas pacientes mais suscetiveis à hipertensão específica da gravidez, mesmo entre aquelas que não desenvolveram hipertensão clinicamente reconhecivel. 
É recomendável que as pacientes que planejam a época do início da procriação sejam esclarecidas com precisão acerca dos riscos a que estão submetidas e que se realizem novos estudos com casuísticas maiores para testar a validade desta associação em gestações múltiplas.

\section{ABSTRACT}

Purpose: to evaluate maternal age of 40 and older as an independent risk factor for pregnancy-induced hypertension (PIH).

Method: we conducted a retrospective cohort study involving analysis of medical records of 2047 women in labor, divided into groups of 298 aged 40 and older and 1749 aged under 40. A multiple logistic regression was done to evaluate the association of maternal age with the occurrence of PIH adjusted by parity, chronic arterial hypertension, diabetes and twin pregnancy.

Results: the incidence of PIH in patients aged 40 and over was $22.1 \%$ (66/298), higher than in patients aged under 40 (16\%, 286/1463). PIH was diagnosed in 27.2\% of primiparous (174/640), 47.6\% of chronic hypertensive (30/ 66) and 27.1\% of diabetic patients (13/48). Advancing maternal age, primiparity and chronic arterial hypertension were associated with the occurrence of $P I H$ in univariate analysis $(O R=1.46,2.58$ and 4.69, respectively). There was no significant association with diabetes. After the adjustment we observed an increase in the strength of the association between maternal age and PIH (adjusted OR =1.69), as well as parity and chronic arterial hypertension.

Conclusion: maternal age of 40 and older was a risk factor for the occurrence of PIH independent of parity, chronic arterial hypertension and diabetes.

KEYWORDS: Pregnancy-induced hypertension. Maternal age. High blood pressure. Diabetes mellitus.

\section{Referências}

1. Dekker GA. Risk factors for preeclampsia. Clin Obstet Gynecol 1999; 42:422-35.

2. Mathews TJ, Hamilton BE. Mean age of mother, 19702000. Natl Vital Stat Rep 2002; 51:1-13.

3. Astolfi P, Zonta LA. Delayed maternity and risk delivery. Paediatr Perinat Epidemiol 2002; 16:67-72.
4. Sibai BM, Ewell M, Levine RJ, et al. Risk factors associated with preeclampsia in healthy nulliparous women.. The Calcium for Preeclampsia Prevention (CPEP) Study Group. Am J Obstet Gynecol 1997; 177:1003-10.

5. Ziadeh S, Yahaya A. Pregnancy outcome at age 40 and older. Arch Gynecol Obstet 2001; 265:30-3.

6. Gregory KD, Korst LM. Age and racial/ethnic differences in maternal, fetal, and placental conditions in laboring patients. Am J Obstet Gynecol 2003; 188:1602-8.

7. van Katwijk C, Peeters LL. Clinical aspects of pregnancy after the age of 35 years: a review of the literature. Hum Reprod Update 1998; 4:185-94.

8. Bertini Oliveira AM, Camano L, Auriemo CR, Novo NF, Feres MC, Delascio D. Diagnóstico do diabetes na gravidez (valores normais da curva glicêmica). Rev Bras Ginecol Obstet 1984; 6:91-100.

9. Abu-Heija AT, Jallad MF, Abukteish F. Maternal and perinatal outcome of pregnancy after age of 45 . J Obstet Gynaecol Res 2000; 26:27-30.

10.Sauer MV, Paulson RJ, Lobo RA. Pregnancy in women 50 or more years of age: outcomes of 22 consecutively established pregnancies from oocyte donation. Fertil Steril 1995; 64:111-5.

11.Vambergue A, Nuttens MC, Goeusse P, Biausque S, Lepeut M, Fontaine P. Pregnancy induced hypertension in women with gestational carbohydrate intolerance: the diagest study. Eur J Obstet Gynecol Reprod Biol 2002; 102:31-5.

12. Sibai B. Pregnancy and coexisting disease hypertension. In: Gabbe SG, Niebyl JR, Simpson JL, editors. Obstetrics: normal and problem pregnancies. $4^{\text {th }}$ ed. New York: Churchill Livingstone; 2002. p.945.

13.Kvetny J, Poulsen HF. Incidence of gestacional hypertension in gestational diabetes mellitus. Arch Gynecol Obstet 2003; 267:153-7.

14.Leonetti G, Lonati L, Cuspidi C. Hypertension and diabetes in women. J Hypertens 2002; 20 (Suppl 2):S23-5.

15.Seoud MA, Nassar AH, Usta IM, Melhem Z, Kazma A, Khalil AM Impact of advanced maternal age on pregnancy outcome. Am J Perinatol 2002; 19:1-8.

16.Hrazdilova O, Unzeitig V, Znojil V, Izakovicova-Holla L, Janku P, Vasku A. Relationship of age and the body mass index to selected hypertensive complications in pregnancy. Int $\mathrm{J}$ Gynaecol Obstet 2001; 75:165-9. 